\title{
The existence of a maximal green sequence is not invariant under quiver mutation
}

\author{
Greg Muller \\ Department of Mathematics \\ University of Michigan \\ Ann Arbor, MI, USA \\ morilac@umich.edu
}

Submitted: Jul 15, 2015; Accepted: May 27, 2016; Published: Jun 10, 2016

Mathematics Subject Classifications: 13F60; 14T05; 16G20

\begin{abstract}
This note provides a specific quiver which does not admit a maximal green sequence, and which is mutation-equivalent to a quiver which does admit a maximal green sequence. This provides a counterexample to the conjecture that the existence of maximal green sequences is invariant under quiver mutation. The proof uses the 'scattering diagrams' of Gross-Hacking-Keel-Kontsevich to show that a maximal green sequence for a quiver determines a maximal green sequence for any induced subquiver.
\end{abstract}

\section{Quivers and maximal green sequences}

The purpose of this paper is to prove that the quiver shown in Figure 1 is a counterexample to the conjecture that the existence of maximal green sequences is invariant under quiver mutation.

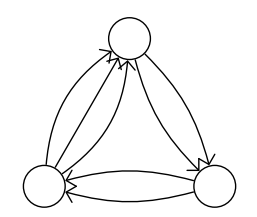

Figure 1: The counterexample quiver

\subsection{Quiver mutation}

A quiver is a finite directed graph without loops or 2-cycles. A quiver Q may be mutated at a vertex $k$ to produce a new quiver $\mu_{k}(\mathrm{Q})$ in three steps. 
1. For each pair of arrows $i \rightarrow k \rightarrow j$ through the vertex $k$, add an arrow $i \rightarrow j$.

2. Reverse the orientation of every arrow incident to $k$.

3. Cancel any directed 2-cycles in pairs.

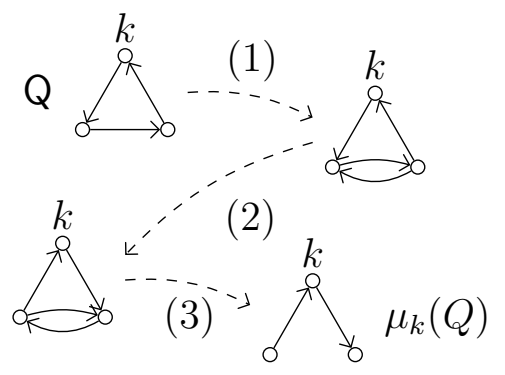

Two quivers are mutation equivalent if there is a sequence of mutations taking one to the other. Mutation equivalence is an equivalence relation, because mutating at the same vertex twice in a row returns to the original quiver.

Remark 1. Quiver mutation was introduced in [FZ02] to encode the combinatorial part of seed mutation in the theory of cluster algebras.

\section{$1.2 \quad g$-vectors}

In this section, we use the Sign Coherence Theorem for quivers to give an elementary definition of $g$-vectors, via $g$-seeds. A $\boldsymbol{g}$-seed is a quiver Q, together with a basis of the lattice $\mathbb{Z}^{N}$ indexed by the vertices of $\mathrm{Q}$. The basis element $g_{k}$ associated to a vertex $k$ is called its $\boldsymbol{g}$-vector.

Example 2. Let $\mathrm{Q}$ be a quiver, and index the vertices of $\mathrm{Q}$ by the numbers $1,2, \ldots, N$. A $g$-seed may be made from $\mathrm{Q}$, where the $g$-vector of the $i$ th vertex is the standard basis element $e_{i}:=(0,0, \ldots, 1, \ldots, 0)$. A $g$-seed of this form is called an initial $\boldsymbol{g}$-seed.

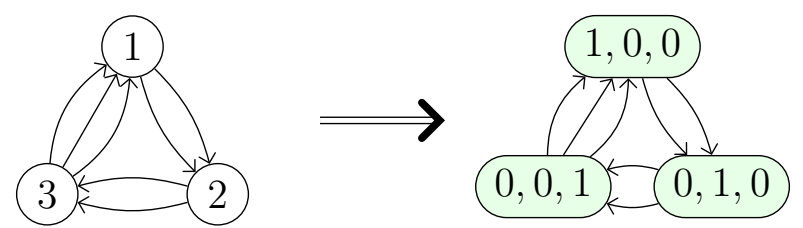

Figure 2: Constructing an initial $g$-seed from an indexed quiver

Given a $g$-seed, any element $v \in \mathbb{Z}^{N}$ may be uniquely expressed as ${ }^{1}$

$$
v=\sum_{\text {vertices } k \text { of } \mathbf{Q}} c_{k}(v) g_{k}, \quad c_{k}(v) \in \mathbb{Z}
$$

A vertex $k$ in a $g$-seed is green if, for all $v \in \mathbb{N}^{N} \subset \mathbb{Z}^{N}$, the coefficient $c_{k}(v) \geqslant 0$. Similarly, a vertex $k$ in a $g$-seed is red if, for all $v \in \mathbb{N}^{N} \subset \mathbb{Z}^{N}$, the coefficient $c_{k}(v) \leqslant 0$. As an example, every vertex in an initial $g$-seed is green.

A $g$-seed may be mutated at a vertex $k$ which is green or red. The underlying quiver mutates as in the previous section, and the $g$-vectors stay the same except at vertex $k$,

\footnotetext{
${ }^{1}$ The coefficient $c_{i}(v)$ is linear on $\mathbb{Z}^{N}$, and given by the dot product with the $c$-vector of vertex $i$.
} 
which changes as follows.

$$
\mu_{k}\left(g_{k}\right):=\left\{\begin{array}{cc}
-g_{k}+\sum_{\forall \text { arrows } j \rightarrow k} g_{j} & \text { if } k \text { is green } \\
-g_{k}+\sum_{\forall \text { arrows } j \leftarrow k} g_{j} & \text { if } k \text { is red }
\end{array}\right\}
$$

Mutation always turns a green vertex into a red vertex and vice versa, so mutating twice in a row at the same vertex returns to the original $g$-seed.

Theorem 3 (Sign coherence, [DWZ10]). After any sequence of mutations of an initial $g$-seed, every vertex in the resulting $g$-seed is either green or red.

Hence, a $g$-seed which is mutation equivalent to an initial $g$-seed may be mutated at an arbitrary sequence of vertices.

Remark 4. Sign coherence was originally conjectured in [FZ07] in the larger generality of skew-symmetrizable matrices. The case of quivers was first proven in [DWZ10], and a categorical proof appeared in [Pla11]. The larger generality of skew-symmetrizable matrices was proven in [GHKK14].

Remark 5. The $g$-vectors of cluster variables with respect to a fixed initial seed were introduced in [FZ07], as the degrees in the universal grading of the cluster algebra with principal coefficients. We have used the Sign Coherence Theorem to simplify the recursive identities (6.12) and (6.13) in [FZ07]; specifically, we have removed the explicit dependence on a choice of initial seed.

\subsection{Maximal green sequences}

Starting with an initial $g$-seed, mutation equivalent $g$-seeds will have all their vertices colored green or red. The following theorem states that monochromatic $g$-seeds in this equivalence class must be unique.

Theorem 6. [BDP14, Prop. 2.10] Let $\left(\mathrm{Q},\left\{g_{k}\right\}\right)$ be a g-seed which is mutation equivalent to an initial g-seed $\left(\mathrm{Q}_{i n},\left\{e_{k}\right\}\right)$.

1. If every vertex of $\left(\mathrm{Q},\left\{g_{k}\right\}\right)$ is green, then there is a quiver isomorphism $f: \mathrm{Q}_{\text {in }} \rightarrow \mathrm{Q}$ such that $g_{f(k)}=e_{k}$.

2. If every vertex of $\left(\mathrm{Q},\left\{g_{k}\right\}\right)$ is red, then there is a quiver isomorphism $f: \mathrm{Q}_{\text {in }} \rightarrow \mathrm{Q}$ such that $g_{f(k)}=-e_{k}$.

While an all-green $g$-seed always exists in such a mutation equivalence class (the initial seed), there may be no all-red $g$-seed. Two examples of such quivers are given in Figure 3 (for proofs, see [BDP14, Prop. 2.21] and [Sev14, Theorem 1]).

Motivated by applications to non-commutative Donaldson-Thomas theory, Keller introduced the idea of a maximal green sequence in [Kel11]. 

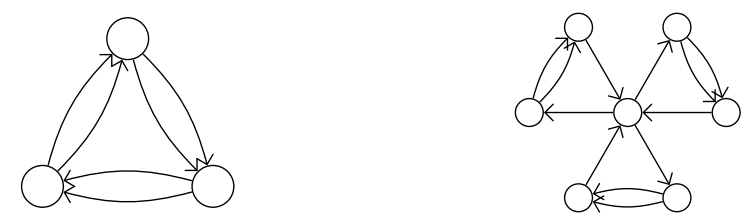

Figure 3: Initial $g$-seeds with these quivers cannot be mutated to an all-red $g$-seed.

Definition 7. A maximal green sequence for a quiver $Q$ is a sequence of mutations starting at an initial $g$-seed $\left(\mathrm{Q},\left\{e_{k}\right\}\right)$, such that

- each mutation is at a green vertex, and

- every vertex in the final $g$-seed is red.

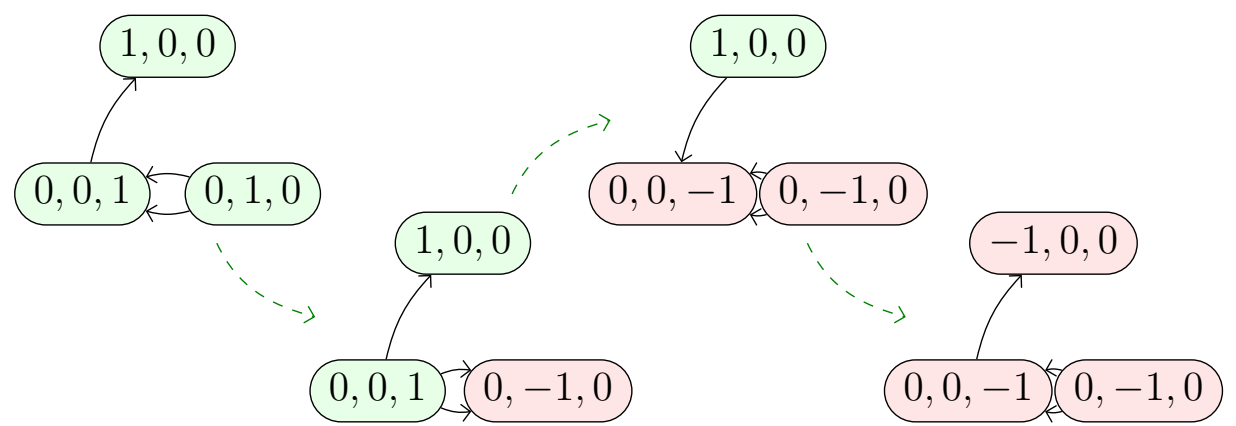

Figure 4: An example of a maximal green sequence

The original purpose of maximal green sequences was to produce identities between products of quantum dilogarithms [Kel11]. However, they have been observed in quivers associated to several families of well-behaved cluster algebras, such as

- acyclic quivers [BDP14, Lemma 2.20],

- quivers from many marked surfaces with boundary $\left[\mathrm{ACC}^{+} 13, \mathrm{Buc14}\right]$, and

- certain quivers defining the coordinate ring of a double Bruhat cell [Yak].

Consequently, it has been conjectured that the existence of a maximal green sequence is equivalent to other good properties of a cluster algebra; for example, that the cluster algebra is equal to its upper cluster algebra. However, since the cluster algebra only depends on the mutation equivalence class of a quiver, these conjectural equivalences implicitly require the following conjecture.

Conjecture 8. If a maximal green sequence exists for a quiver $Q$, then a maximal green sequence exists for any mutation equivalent quiver $Q^{\prime}$.

One purpose of this note is to provide a counterexample to this conjecture. 


\subsection{Induced subquivers}

Given a subset $V$ of the vertices of a quiver $Q$, the induced subquiver $\mathrm{Q}^{\dagger}$ is the quiver with vertex set $V$ and arrow set consisting of arrows in $\mathrm{Q}$ between pairs of vertices in $V$.

The key tool in this note is the following theorem.

Theorem 9. If a quiver $\mathrm{Q}$ admits a maximal green sequence, then any induced subquiver $\mathrm{Q}^{\dagger}$ admits a maximal green sequence. If a maximal green sequence for $\mathrm{Q}$ begins with a sequence of mutations on vertices in $\mathrm{Q}^{\dagger}$, then there is a maximal green sequence for $\mathrm{Q}^{\dagger}$ which begins with the same sequence of mutations.

The proof of theorem uses the theory of scattering diagrams, developed in [GPS10, KS13, GHK15] and connected to cluster algebras in [GHKK14]. Since the relevant theory is elaborate and self-contained, the proof of Theorem 9 will be deferred to Section 5 .

\section{Existence and non-existence of maximal green sequences}

This section collects several results about maximal green sequences, culminating in the promised counterexample.

\subsection{Acyclic quivers}

A quiver is acyclic if there are no directed cycles. Acyclic quivers are the most straightforward class of quivers with a maximal green sequence.

Proposition 10. [BDP14, Lemma 2.20] An acyclic quiver $\mathrm{Q}$ admits a maximal green sequence.

In fact, a maximal green sequence can always be given by iteratively mutating at source vertices which have not yet been mutated.

\subsection{Quivers with 2 vertices}

Let $b$ be a positive integer, and let $\mathrm{Q}_{b}$ denote the quiver on two vertices with $b$-many arrows from 1 to 2 (Figure 5).

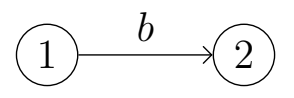

Figure 5: The quiver $\mathrm{Q}_{b}$

Every quiver of the form $Q_{b}$ is acyclic, and thus it admits a maximal green sequence by iteratively mutating at sources. The following lemma asserts that this is the only maximal green sequence. 
Lemma 11. If $b \geqslant 2$, then the only maximal green sequence for $\mathrm{Q}_{b}$ is mutation at vertex 1 and then vertex 2 .

Proof. It is easy to check that mutation at 1 and then 2 is a maximal green sequence, and that it is the only maximal green sequence beginning with mutation at 1 .

Consider the mutation of an initial $g$-seed with quiver $\mathrm{Q}_{b}$ at 2 (Figure 6).

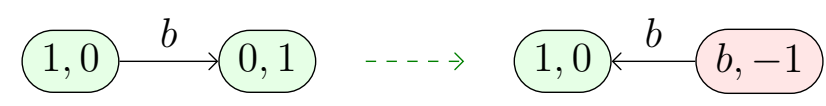

Figure 6: Mutating an initial seed at vertex 2

Define polynomials $F_{i}$ of $b$ for all $i \in \mathbb{N}$ recursively, by ${ }^{2}$

$$
\begin{gathered}
F_{0}=0, \quad F_{1}=1 \\
F_{i+1}=b F_{i}-F_{i-1}
\end{gathered}
$$

We now make the following claim. For $i \geqslant 1$, after a sequence of $i$-many green mutation starting at 2, the resulting $g$-seed is isomorphic to the $g$-seed in Figure 7 .

$$
F_{i},-F_{i-1}<F_{i+1},-F_{i}
$$

Figure 7: The $g$-seed after $i$ green mutations starting at 2

Figure 6 establishes the claim for $i=1$. Assume the claim holds for $i$-many mutations starting at 2 . The $g$-seed in Figure 7 has a unique green vertex, so a sequence of $(i+1)$ many green mutations starting at 2 must come from mutation the this seed at the green vertex. The resulting seed, shown in Figure 8, is of the desired form.

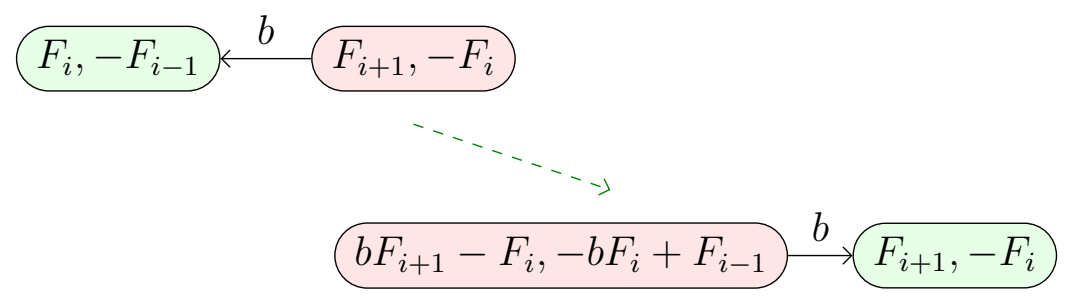

Figure 8: Mutating the $g$-seed in Figure 7 at the green vertex

By induction, the claim holds for all $i$. In particular, no sequence of green mutations starting at 2 produces a $g$-seed with all vertices red, and so there are no maximal green sequences starting at 2 .

\footnotetext{
${ }^{2} \mathrm{Up}$ to reindexing and a change of variables, these are the Chebyshev polynomials of the second kind.
} 


\subsection{Quivers with 3 vertices}

Let $a, b, c$ be non-negative integers, and let $\mathrm{Q}_{a, b, c}$ denote the quiver on 3 vertices with $a$-many arrows from 1 to $2, b$-many arrows from 2 to 3 , and $c$-many arrows from 3 to 1 (Figure 9).

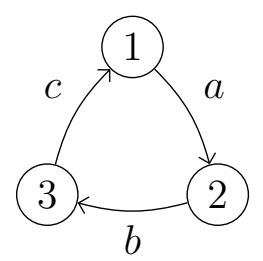

Figure 9: The quiver $Q_{a, b, c}$

Theorem 12. If $a, b, c \geqslant 2$, then the quiver $\mathrm{Q}_{a, b, c}$ does not admit a maximal green sequence.

Proof. Assume there is a maximal green sequence which starts by mutating at the vertex 2. By Theorem 9, this determines a maximal green sequence for the induced subquiver on the vertices $\{1,2\}$ which starts by mutating at vertex 2 , which is impossible by Lemma 11. By symmetric arguments, a maximal green sequence cannot start with 1 or 3 .

However, there are many quivers of the form $Q_{a, b, c}$ which are mutation equivalent to an acyclic quiver.

Lemma 13. [BBH11, Theorem 1.1] The quiver $\mathrm{Q}_{a, b, c}$ is mutation-equivalent to an acyclic quiver if and only if

$$
a b c-a^{2}-b^{2}-c^{2}+4<0 \text { or } \min (a, b, c)<2
$$

Combining the last two results provides many counter-examples to Conjecture 8.

Corollary 14. Let $a, b, c \geqslant 2$ with $a b c-a^{2}-b^{2}-c^{2}+4<0$. Then $\mathrm{Q}_{a, b, c}$ does not admit a maximal green sequence, but is mutation equivalent to a quiver which admits a maximal green sequence.

The simplest such quiver is $Q_{2,2,3}$. For the benefit of the reader, Figure 10 shows a sequence of quiver mutations relating $Q_{2,2,3}$ to the acyclic quiver $Q_{0,2,1}$, and Figure 4 shows a maximal green sequence for quiver $Q_{0,2,1}$.

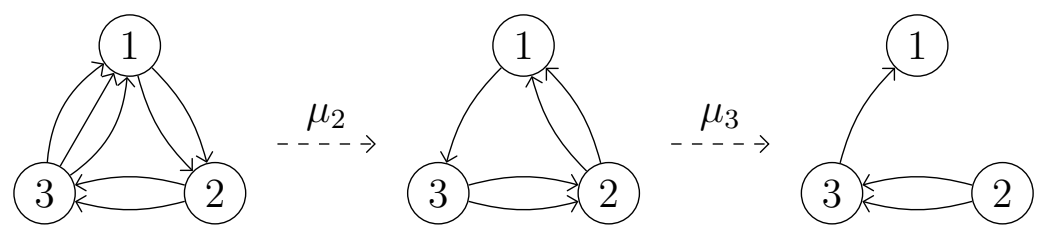

Figure 10: The quiver $Q_{2,2,3}$ is mutation equivalent to $Q_{0,2,1}$ 


\section{Green-to-red sequences}

\subsection{Green-to-red sequences}

This section considers a weaker property than the existence of a maximal green sequence, which has some of the properties expected of maximal green sequences.

Definition 15. A green-to-red sequence for a quiver $Q$ is a sequence of mutations which takes an initial $g$-seed with quiver $\mathrm{Q}$ to a $g$-seed with all vertices red.

By Theorem 6, the final $g$-seed in a green-to-red sequence has quiver Q, and all $g$-vectors of the form $-e_{k}$. The existence of a green-to-red sequence is equivalent to the existence of an all-red $g$-seed which is mutation-equivalent to an initial $g$-seed on Q.

A maximal green sequence is clearly a green-to-red sequence. However, a quiver may admit a green-to-red sequence but not admit a maximal green sequence. In particular, $\mathrm{Q}_{2,2,3}$ admits a green-to-red sequence (Figure 11).
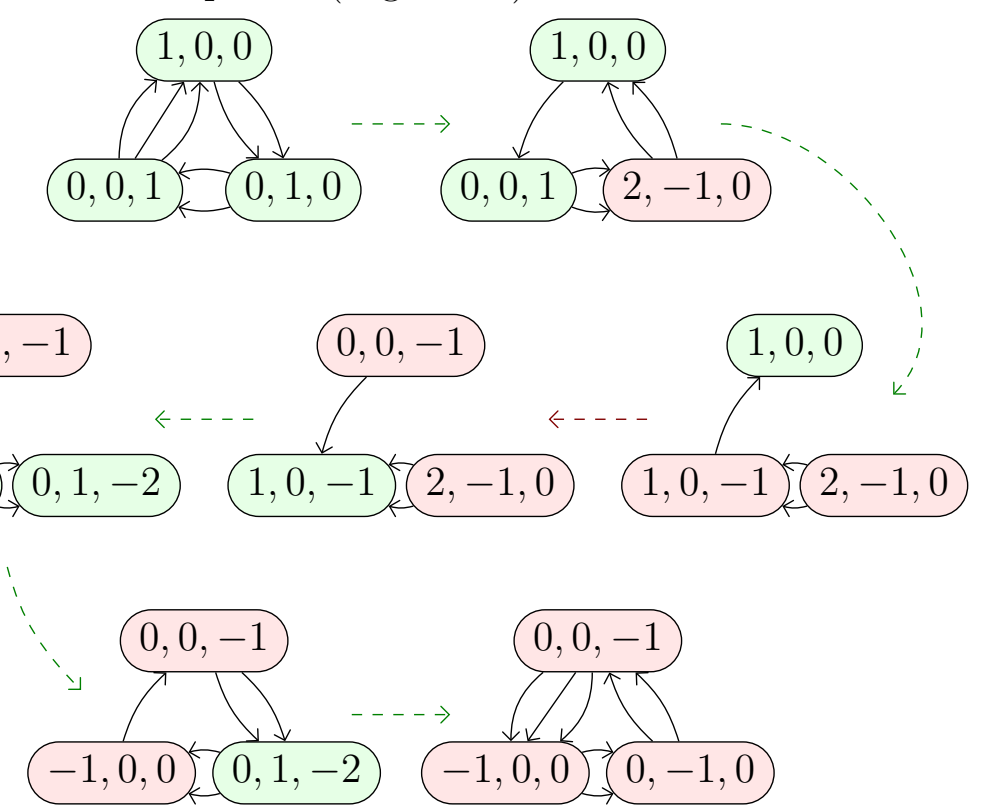

Figure 11: The quiver $Q_{2,2,3}$ has green-to-red sequence

Remark 16. An equivalent condition to the existence of a green-to-red sequence appears in [GHKK14, Prop.8.26], together with important consequences for the scattering diagram (see Section 4.4 for the connection).

Like maximal green sequences, the existence of a green-to-red sequence is preserved under passing to an induced subquiver.

Theorem 17. If a quiver $\mathrm{Q}$ admits a green-to-red sequence, then any induced subquiver $\mathrm{Q}^{\dagger}$ admits a green-to-red sequence.

The proof appears in Section 5. 


\subsection{Conjugation}

By Theorem 6, the quivers at the beginning and end of a green-to-red sequence are canonically identified. This allows us to conjugate a green-to-red sequence for a quiver $\mathrm{Q}$ by any sequence of mutations from $\mathrm{Q}$ to another quiver $\mathrm{Q}^{\prime}$.

Theorem 18. The conjugation of a green-to-red sequence by any other sequence of mutations is a green-to-red sequence.

Theorem 18 will be proved using scattering diagrams in Section 5. As a corollary, the existence of green-to-red sequences is invariant under mutation.

Corollary 19. If a green-to-red sequence exists for a quiver $\mathrm{Q}$, then a green-to-red sequence exists for any mutation equivalent quiver $\mathrm{Q}^{\prime}$.

\section{Scattering diagrams}

The remainder of this note reviews the theory of scattering diagrams and the proofs of the necessary theorems. The main references are [GPS10, KS13, GHK15, GHKK14].

\subsection{Formal elementary transformations}

Let $\mathrm{Q}$ be a quiver, and index the vertices of $\mathrm{Q}$ with the set $\{1,2, \ldots, r\}$. The quiver may be encoded in the skew-adjacency matrix $\mathrm{B}$, which is the following $r \times r$ matrix.

$$
\mathrm{B}_{i j}=(\# \text { of arrows from } j \text { to } i)-(\# \text { of arrows from } i \text { to } j)
$$

Consider the ring

$$
R:=\mathbb{Q}\left[x_{1}^{ \pm 1}, x_{2}^{ \pm 1}, \ldots, x_{r}^{ \pm 1}\right]\left[\left[y_{1}, y_{2}, \ldots, y_{r}\right]\right]
$$

that is, formal power series in the variables $y_{1}, y_{2}, \ldots, y_{r}$ with coefficients in the ring of Laurent polynomials in $x_{1}, x_{2}, \ldots, x_{r}$. We use multinomial notation for the $x$ and $y$ variables; that is,

$$
\forall m=\left(m_{1}, m_{2}, \ldots, m_{r}\right) \in \mathbb{Z}^{r}, \quad x^{m}:=\prod_{i=1}^{r} x_{i}^{m_{i}}, \quad \forall n=\left(n_{1}, n_{2}, \ldots, n_{r}\right) \in \mathbb{N}^{r}, \quad y^{n}:=\prod_{i=1}^{r} y_{i}^{n_{r}}
$$

For $n \in \mathbb{N}^{r}$, define the formal elementary transformation $E_{n}: R \longrightarrow R$ by

$$
E_{n}\left(x^{m}\right)=\left(1+x^{\mathrm{B} n} y^{n}\right)^{\frac{n \cdot m}{\operatorname{gcd}(n)}} x^{m}, \quad E_{n}\left(y^{n^{\prime}}\right)=y^{n^{\prime}}
$$

Here, $n \cdot m=\sum_{i} n_{i} m_{i}$ denotes the Euclidean inner product, and $\operatorname{gcd}(n)$ is the greatest common divisor of the coordinates of $n$. This is an automorphism, with inverse

$$
E_{n}^{-1}\left(x^{m}\right)=\left(1+x^{\mathrm{B} n} y^{n}\right)^{-\frac{n \cdot m}{\operatorname{gcd}(n)}} x^{m}, \quad E_{n}^{-1}\left(y^{n^{\prime}}\right)=y^{n^{\prime}}
$$

Formal elementary transformations need not commute, with the following basic example. 
Proposition 20. Let $n, n^{\prime} \in \mathbb{N}^{r}$ with $n \cdot \mathrm{B} n^{\prime}=1$. Then

$$
E_{n} E_{n^{\prime}}=E_{n^{\prime}} E_{n+n^{\prime}} E_{n}
$$

Proof. Since B is skew-symmetric, $n \cdot \mathrm{B} n=n^{\prime} \cdot \mathrm{B} n^{\prime}=0$ and $n^{\prime} \cdot \mathrm{B} n=-n \cdot \mathrm{B} n^{\prime}=-1$. Furthermore, since $n \cdot \mathrm{B} n^{\prime}=1$, both $\operatorname{gcd}(n)=\operatorname{gcd}\left(n^{\prime}\right)=1$. In a similar argument, $\left(n+n^{\prime}\right) \cdot \mathrm{B} n^{\prime}=n^{\prime} \cdot \mathrm{B} n^{\prime}+n \cdot \mathrm{B} n^{\prime}=1$, and so $\operatorname{gcd}\left(n+n^{\prime}\right)=1$. We now compute the two actions directly.

$$
\begin{aligned}
E_{n} E_{n^{\prime}}\left(x^{m}\right) & =E_{n}\left(\left(1+x^{\mathrm{B} n^{\prime}} y^{n^{\prime}}\right)^{n^{\prime} \cdot m} x^{m}\right) \\
& =\left(1+\left(1+x^{\mathrm{B} n} y^{n}\right)^{n \cdot B n^{\prime}} x^{\mathrm{B} n^{\prime}} y^{n^{\prime}}\right)^{n^{\prime} \cdot m}\left(1+x^{\mathrm{B} n} y^{n}\right)^{n \cdot m} x^{m} \\
& =\left(1+x^{\mathrm{B} n^{\prime}} y^{n^{\prime}}+x^{\mathrm{B}\left(n+n^{\prime}\right)} y^{n+n^{\prime}}\right)^{n^{\prime} \cdot m}\left(1+x^{\mathrm{B} n} y^{n}\right)^{n \cdot m} x^{m}
\end{aligned}
$$

For comparison, $E_{n^{\prime}} E_{n+n^{\prime}} E_{n}\left(x^{m}\right)$ is equal to

$$
\begin{aligned}
& =E_{n^{\prime}} E_{n+n^{\prime}}\left(\left(1+x^{\mathrm{B} n} y^{n}\right)^{n \cdot m} x^{m}\right) \\
& =E_{n^{\prime}}\left(\left(1+\left(1+x^{\mathrm{B}\left(n+n^{\prime}\right)} y^{n+n^{\prime}}\right)^{-1} x^{\mathrm{B} n} y^{n}\right)^{n \cdot m}\left(1+x^{\mathrm{B}\left(n+n^{\prime}\right)} y^{n+n^{\prime}}\right)^{n \cdot m+n^{\prime} \cdot m} x^{m}\right) \\
& =E_{n^{\prime}}\left(\left(1+x^{\mathrm{B}\left(n+n^{\prime}\right)} y^{n+n^{\prime}}+x^{\mathrm{B} n} y^{n}\right)^{n \cdot m}\left(1+x^{\mathrm{B}\left(n+n^{\prime}\right)} y^{n+n^{\prime}}\right)^{n^{\prime} \cdot m} x^{m}\right) \\
& =E_{n^{\prime}}\left(\left(1+\left(1+x^{\mathrm{B} n^{\prime}} y^{n^{\prime}}\right) x^{\mathrm{B} n} y^{n}\right)^{n \cdot m}\left(1+x^{\mathrm{B}\left(n+n^{\prime}\right)} y^{n+n^{\prime}}\right)^{n^{\prime} \cdot m} x^{m}\right) \\
& =\left(1+x^{\mathrm{B} n} y^{n}\right)^{n \cdot m}\left(1+\left(1+x^{\mathrm{B} n^{\prime}} y^{n^{\prime}}\right)^{-1} x^{\mathrm{B}\left(n+n^{\prime}\right)} y^{n+n^{\prime}}\right)^{n^{\prime} \cdot m}\left(1+x^{\mathrm{B} n^{\prime}} y^{n^{\prime}}\right)^{n^{\prime} \cdot m} x^{m} \\
& =\left(1+x^{\mathrm{B} n} y^{n}\right)^{n \cdot m}\left(1+x^{\mathrm{B} n^{\prime}} y^{n^{\prime}}+x^{\mathrm{B}\left(n+n^{\prime}\right)} y^{n+n^{\prime}}\right)^{n^{\prime} \cdot m} x^{m}
\end{aligned}
$$

Since both actions send $y^{n}$ to $y^{n}$, they coincide on a generating set.

Remark 21. Following ideas of Reineke [Rei10] and Kontsevich-Soibelman [KS11], any such identity between formal elementary transformations determines an analogous identity among quantum dilogarithms [Kel11]. The above proposition corresponds to the Faddeev-Kashaev identity, which in turn implies the pentagonal identity for the Rogers' dilogarithm.

\subsection{Scattering diagrams}

Scattering diagrams may be regarded as a method for visualizing diagrams of formal elementary transformations. For a quiver $\mathrm{Q},{ }^{3}$ a wall is a pair $(n, W)$ consisting of

- a non-zero element $n \in \mathbb{N}^{r}$, and

- a convex polyhedral cone $W$ in $\mathbb{R}^{r}$ which spans $n^{\perp}:=\left\{m \in \mathbb{R}^{r} \mid n \cdot m=0\right\}$.

\footnotetext{
${ }^{3}$ While $\mathrm{Q}$ does not appear in the definition of a wall, it is necessary to associate a formal elementary transformation $E_{n}$ to the wall, and so we shouldn't consider walls without first having a quiver in mind.
} 
Since $n \in \mathbb{N}^{r}$, this implies that $W \cap\left(\mathbb{R}_{>0}\right)^{r}=W \cap\left(\mathbb{R}_{<0}\right)^{r}=\emptyset$. We will refer to the open half-space $\left\{m \in \mathbb{R}^{r} \mid n \cdot m>0\right\}$ as the green side of $W$, and $\left\{m \in \mathbb{R}^{r} \mid n \cdot m<0\right\}$ as the red side of $W$.

A scattering diagram $\mathfrak{D}$ is a collection of walls in $\mathbb{R}^{r}$ for the same $Q .{ }^{4}$ Let us say a smooth path $p:[0,1] \rightarrow \mathbb{R}^{r}$ in a scattering diagram $\mathfrak{D}$ is finite transverse if

- $p(0)$ and $p(1)$ are not in any walls,

- whenever the image of $p$ intersects a wall, it crosses it transversely, and

- the image of $p$ intersects finitely many walls, and does not intersect the boundary of a wall or the intersection of two walls which span different hyperplanes.

A finite transverse path $p$ determines an automorphism of $R$, as follows. List the walls crossed by $p$ in order: ${ }^{5}$

$$
\left(n_{1}, W_{1}\right),\left(n_{2}, W_{2}\right), \ldots,\left(n_{k}, W_{k}\right)
$$

and to each, associate the sign

$$
\epsilon_{i}:=\left\{\begin{array}{ll}
+1 & \text { if } p \text { crossed } W_{i} \text { from the green side to the red side } \\
-1 & \text { if } p \text { crossed } W_{i} \text { from the red side to the green side }
\end{array}\right\}
$$

Then the path-ordered product of $p$ is

$$
E_{n_{k}}^{\epsilon_{k}} E_{n_{k-1}}^{\epsilon_{k-1}} \cdots E_{n_{1}}^{\epsilon_{1}}
$$

A finite scattering diagram is consistent if the path-ordered product associated to every finite transverse loop is the trivial automorphism of $R$. Two finite scattering diagrams for the same quiver are equivalent if any path which is finite transitive in both diagrams determines the same path-ordered product. ${ }^{6}$

Remark 22. A finite scattering diagram determines a morphism diagram whose objects are a copy of $R$ for each chamber, and whose arrows are elementary transformations for each wall between chambers. The consistency condition is equivalent to the commutativity of this diagram.

Example 23. Consider the quiver $\mathrm{Q}_{1}$ in Figure 12a.

We claim the scattering diagram $\mathfrak{D}$ in Figure $12 \mathrm{c}$ is consistent. The path-ordered product of the simple transverse path $p$ in the figure is

$$
E_{(0,1)}^{-1} E_{(1,1)}^{-1} E_{(1,0)}^{-1} E_{(0,1)} E_{(1,0)}
$$

By Proposition 20, this is the trivial automorphism of $R$. All other path-ordered products of finite transverse loops are conjugations of this expression, and so they are also trivial. ${ }^{7}$ Hence, $\mathfrak{D}$ is a consistent scattering diagram.

\footnotetext{
${ }^{4}$ We explicitly allow multiple copies of the same wall.

${ }^{5}$ The path $p$ may simultaneously cross multiple walls, but only if those walls span the same hyperplane. In this case, the walls may be listed in any order, since the corresponding automorphisms commute.

${ }^{6}$ Equivalence of scattering diagrams is a fairly restrictive condition; essentially, it just allows walls to be split into multiple walls, and vice versa.

${ }^{7}$ Whenever $r=2$, checking the any finite transverse transverse loop around the origin suffices to check consistency.
} 

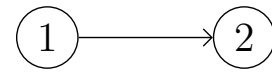

(a) The quiver $Q_{1}$

$\mathrm{B}=\left[\begin{array}{cc}0 & -1 \\ 1 & 0\end{array}\right]$

(b) The matrix B
$((1,0),(0, \mathbb{R}))$

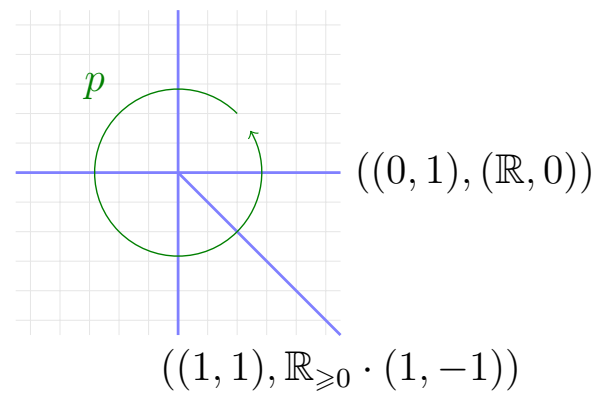

(c) The scattering diagram $\mathfrak{D}$

Figure 12: An example of a consistent finite scattering diagram

Example 24. Consider the quiver $\mathrm{Q}_{1,1,1}$ in Figure 13a. Since $r=3$, a scattering diagram for $Q_{1,1,1}$ consists of scale-invariant subsets of $\mathbb{R}^{3}$, it may be visualized by intersecting with a unit sphere and stereographically projecting onto the plane $x_{1}+x_{2}+x_{3}=\sqrt{3}$.

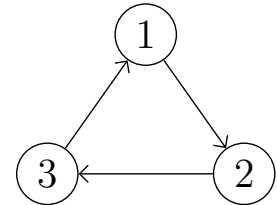

(a) The quiver $Q_{1,1,1}$

$$
B=\left[\begin{array}{ccc}
0 & -1 & 1 \\
1 & 0 & -1 \\
-1 & 1 & 0
\end{array}\right]
$$

(b) The matrix B

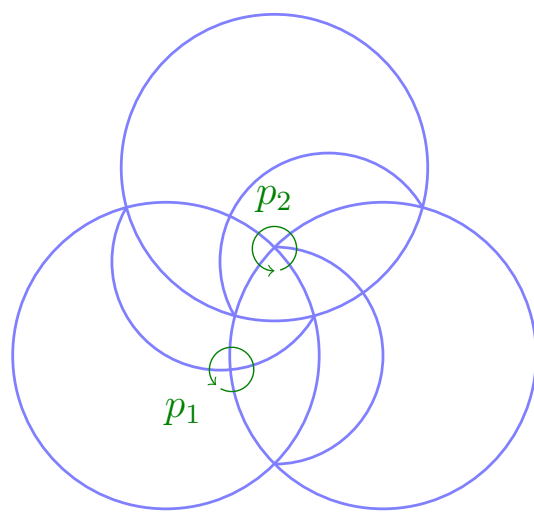

(c) Stereographic projection of the scattering diagram $\mathfrak{D}$

Figure 13: An example of a consistent finite scattering diagram

The scattering diagram $\mathfrak{D}$ in Figure 13c is consistent. Checking this can be reduced to checking the path-ordered product of tiny loops around intersections of walls. The intersections among the walls in $\mathfrak{D}$ are the nine rays which project to the the nine intersections in Figure 13c. The triviality of the tiny loops around the 4-valent intersections (such as $p_{1}$ in the figure) is the commutativity of the associated elementary transformations, while the triviality of the tiny loops around the 5-valent intersections (such as $p_{2}$ in the figure) reduces to Proposition 20. 


\subsection{Reduction of scattering diagrams}

The scattering diagrams we are interested in typically have infinitely many walls, and so there are not enough finite transverse loops to test for the 'right' notion of consistency. The simplest solution is to make sense of formal limits of consistent finite scattering diagrams.

Let $I$ be a monomial ideal in $\mathbb{Q}\left[\left[y_{1}, y_{2}, \ldots, y_{r}\right]\right]$. For each $n \in \mathbb{N}^{r}, E_{n}$ and $E_{n}^{-1}$ descend to well-defined automorphisms of $R / I$, which are trivial when $y^{n} \in I$. A finite scattering diagram is consistent mod $\boldsymbol{I}$ if the path-ordered product associated to every transverse loop is the trivial automorphism of $R / I$. Two finite scattering diagrams for the same quiver are equivalent mod $\boldsymbol{I}$ if any path which is finite transverse in both induces the same automorphism of $R / I$.

The reduction $\mathfrak{D} / I$ of a scattering diagram $\mathfrak{D}$ is obtained by deleting any wall of the form $(n, W)$ with $y^{n} \in I$. If $\mathfrak{D}$ is a consistent finite scattering diagram, then $\mathfrak{D} / I$ is consistent mod $I$. This idea can be extended to a criterion for the consistency of infinite scattering diagrams.

A scattering diagram $\mathfrak{D}$ is consistent if, for each monomial ideal $I \subset \mathbb{Q}\left[\left[y_{1}, y_{2}, \ldots, y_{r}\right]\right]$ with finite dimensional quotient, the reduction $\mathfrak{D} / I$ is finite and consistent mod $I$. Two scattering diagrams $\mathfrak{D}_{1}$ and $\mathfrak{D}_{2}$ for the same quiver are equivalent if, for every monomial ideal $I \subset \mathbb{Q}\left[\left[y_{1}, y_{2}, \ldots, y_{r}\right]\right]$ with finite dimensional quotient, the reductions $\mathfrak{D}_{1} / I$ and $\mathfrak{D}_{2} / I$ are finite and equivalent mod $I$.

We may now state one of the main existence and uniqueness results of the theory.

Theorem 25. [GHKK14, Theorems 1.13 and 1.28] For each quiver $\mathrm{Q}$, there is a consistent scattering diagram $\mathfrak{D}(\mathrm{Q})$, unique up to equivalence, such that

- for each $i \in\{1,2, \ldots, r\}$, there is a wall of the form $\left(e_{i}, e_{i}^{\perp}\right),{ }^{8}$ and

- every other wall $(n, W)$ in $\mathfrak{D}(\mathrm{Q})$ has the property that $\mathrm{B} n \notin W$.

Remark 26. [GHKK14] prove a more general theorem, which states that, given a scattering diagram $\mathfrak{D}_{\text {in }}$ which is consistent modulo $I$, there is a unique (up to equivalence) consistent scattering diagram $\mathfrak{D}$ such that $\mathfrak{D} / I=\mathfrak{D}_{\text {in }}$, and $\mathfrak{D} \backslash \mathfrak{D}_{\text {in }}$ consists of walls $(n, W)$ such that $\mathrm{B} n \notin W$. Theorem 25 is the case where $\mathfrak{D}$ is the scattering diagram consisting of the coordinate hyperplanes, which is consistent modulo $\left\langle y_{1}, y_{2}, \ldots, y_{r}\right\rangle^{2}$.

Example 27. Consider the quiver $\mathrm{Q}_{2}$ in Figure 14a.

The scattering diagram in Figure 14 is consistent. This fact is equivalent to the following infinite product identity, which is proven in [GMN10, Equation A.6].

$$
E_{n_{1}} E_{n_{2}}=E_{n_{2}} E_{n_{1}+2 n_{2}} E_{2 n_{1}+3 n_{2}} \cdots\left(\prod_{k=0}^{\infty} E_{2^{k}\left(n_{1}+n_{2}\right)}\right)^{2} \cdots E_{3 n_{1}+2 n_{2}} E_{2 n_{1}+n_{2}} E_{n_{1}}
$$

The only walls $(n, W)$ such that $\mathrm{B} n \in W$ are the coordinate hyperplanes; hence, this scattering diagram is the unique (up to equivalence) scattering diagram $\mathfrak{D}\left(\mathrm{Q}_{2}\right)$.

\footnotetext{
${ }^{8}$ That is, the hyperplane where the $i$ th coordinate vanishes is a wall.
} 


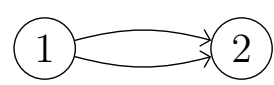

(a) The quiver $Q_{2}$

$\mathrm{B}=\left[\begin{array}{cc}0 & -2 \\ 2 & 0\end{array}\right]$

(b) The matrix B

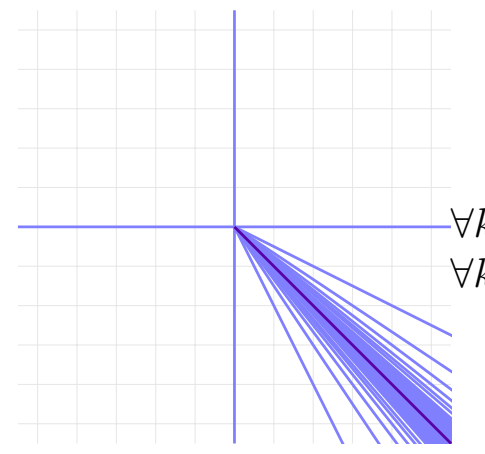

(c) The scattering diagram $\mathfrak{D}\left(\mathrm{Q}_{2}\right)$
Walls:

$$
\left(e_{1}, e_{1}^{\perp}\right)=((1,0),(0, \mathbb{R}))
$$$$
\left(e_{2}, e_{2}^{\perp}\right)=((0,1),(\mathbb{R}, 0))
$$

$\forall k \geqslant 1,\left((k, k+1), \mathbb{R}_{\geqslant 0} \cdot(k+1,-k)\right)$

$\forall k \geqslant 1,\left((k+1, k), \mathbb{R}_{\geqslant 0} \cdot(k,-k-1)\right)$

For each $k \geqslant 0$, two copies of

$$
\left(\left(2^{k}, 2^{k}\right), \mathbb{R}_{\geqslant 0} \cdot(1,-1)\right)
$$

Figure 14: An example of a consistent infinite scattering diagram

Example 28. Consider the quiver $\mathrm{Q}_{1,1,2}$ in Figure 15a. Since $r=3$, a scattering diagram for $Q_{1,1,2}$ consists of scale-invariant subsets of $\mathbb{R}^{3}$, it may be visualized by intersecting with a unit sphere and stereographically projecting onto the plane $x_{1}+x_{2}+x_{3}=\sqrt{3}$ (Figure 15c). In the figure, the purple arc is the projection of a half-plane which supports an infinite number of walls.

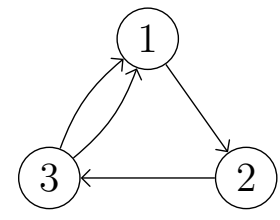

(a) The quiver $Q_{1,1,2}$

$$
B=\left[\begin{array}{ccc}
0 & -1 & 2 \\
1 & 0 & -1 \\
-2 & 1 & 0
\end{array}\right]
$$

(b) The matrix B

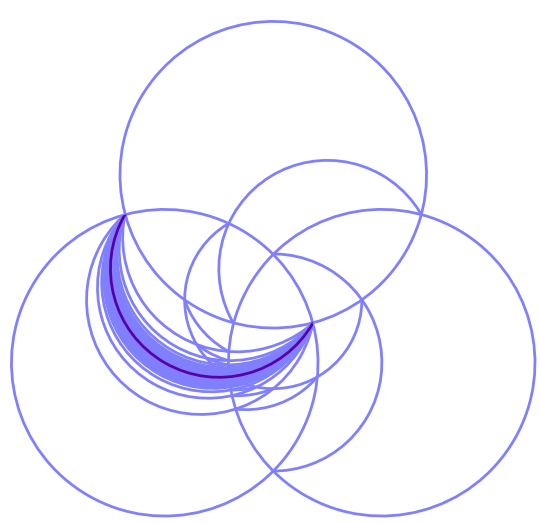

(c) Stereographic projection of the scattering diagram $\mathfrak{D}\left(\mathrm{Q}_{1,1,2}\right)$

Figure 15: An example of a consistent infinite scattering diagram

The scattering diagram $\mathfrak{D}$ in Figure $15 \mathrm{c}$ is consistent. Again, checking this can be reduced to checking the path-ordered product of tiny loops around intersections of walls. All but three of the intersections are 5-valent, and correspond to Proposition 20. There is an intersection of an infinite number of walls at either end of the purple wall; the consistency around these intersections reduces to the consistency of Figure 13c. There is 
one final intersection between the purple ray and two blue walls; the formal elementary transformations of these walls all commute with each other, which implies consistency.

\subsection{Connection to $g$-vectors}

One remarkable feature of the scattering diagram $\mathfrak{D}(\mathrm{Q})$ is that it encodes every $g$-seed mutation equivalent to the initial seed on $Q$. A chamber in a scattering diagram $\mathfrak{D}$ is a path-connected component of the complement $\mathbb{R}^{r}-\mathfrak{D}$.

Example 29. Since no walls can pass through $\left(\mathbb{R}_{>0}\right)^{r}$ and $\left(\mathbb{R}_{<0}\right)^{r}$ and the coordinate hyperplanes are walls in $\mathfrak{D}(\mathrm{Q})$, it follows that the all-positive chamber $\left(\mathbb{R}_{>0}\right)^{r}$ and the all-negative chamber $\left(\mathbb{R}_{<0}\right)^{r}$ must be chambers in $\mathfrak{D}(\mathrm{Q})$.

Let us call a chamber in $\mathfrak{D}(\mathrm{Q})$ reachable if it can be connected to the all-positive chamber by a finite transverse path.

Theorem 30. [GHKK14, Lemma 2.9] Let $\left\{g_{1}, g_{2}, \ldots, g_{r}\right\}$ be the g-vectors in a g-seed which is mutation-equivalent to the initial g-seed ${ }^{9}$ with quiver $\mathrm{Q}$. Then

$$
\mathbb{R}_{>0} g_{1}+\mathbb{R}_{>0} g_{2}+\ldots+\mathbb{R}_{>0} g_{r}
$$

is a reachable chamber in $\mathfrak{D}(\mathrm{Q})$. This induces a bijection between g-seeds mutation equivalent to the initial $g$-seed on $\mathrm{Q}$, and reachable chambers of $\mathfrak{D}(\mathrm{Q})$.

The theorem allows us to translate statements about $g$-seeds into statements about reachable chambers. In what follows, by ' $g$-seed', we mean a $g$-seed mutation-equivalent to the initial seed on $\mathrm{Q}$.

- A vertex $k$ in a $g$-seed is green if and only if the $g$-vector $g_{k}$ is on the green side of the wall spanned by the other $g$-vectors.

- Two $g$-seeds are related by mutation if and only the corresponding reachable chambers share a facet. The green mutation goes from the green side to the red side, and the red mutation goes from the red side to the green side.

- A $g$-seed with all green vertices must correspond to the all-positive chamber, and so it must be the initial $g$-seed.

- A $g$-seed with all red vertices must correspond to the all-negative chamber. Hence, the existence of an all-red seed is equivalent to the all-negative chamber being reachable.

A finite transverse path $p$ which begins in a reachable chamber defines a sequence of mutations of the corresponding $g$-seed, and every sequence of mutations of a $g$-seed

\footnotetext{
${ }^{9}$ Since the vertices of $\mathrm{Q}$ are already indexed by $\{1,2, \ldots, r\}$, we use the initial seed corresponding to this indexing.
} 
can be encoded into a finite transverse path. ${ }^{10}$ Under this equivalence, the sequences of mutations we are interested in correspond to certain finite transverse paths in $\mathfrak{D}(\mathbf{Q})$.

- A maximal green sequence is equivalent to a finite transitive path from the allpositive chamber to the all-negative chamber, which always crosses a wall from the green side to the red side.

- A green-to-red sequence is equivalent to a finite transitive path from the all-positive chamber to the all-negative chamber.

Hence, the existence of a green-to-red sequence on $Q$ is equivalent to the all-negative chamber being reachable in $\mathfrak{D}(\mathrm{Q})$.

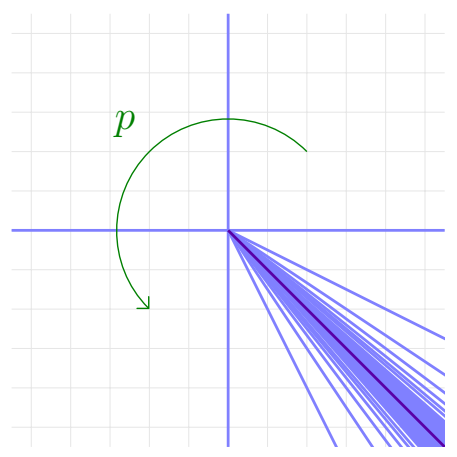

(a) A path

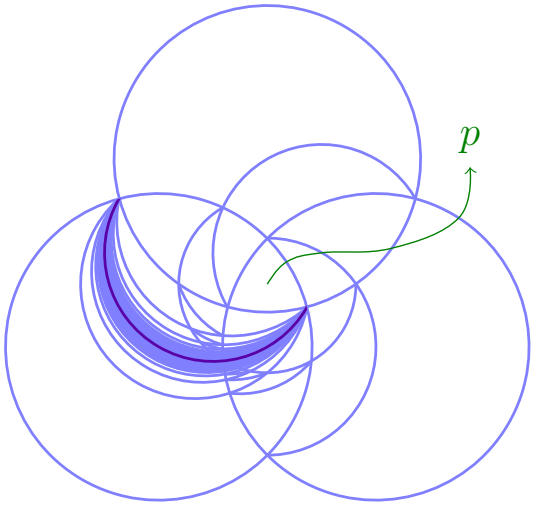

(b) Stereographic projection of a path

Figure 16: Paths corresponding to maximal green sequences

Example 31. For $\mathrm{Q}_{2}$ as in Figure $14 \mathrm{a}$, there is a unique maximal green sequence, which can be realized by the path $p$ in Figure 16a.

The scattering diagram in Figure 16a also gives a visual proof of Lemma 11. A finite transverse path cannot cross the purple ray, since it supports an infinite number of walls. ${ }^{11}$ If a maximal green sequence could begin by mutating at vertex 2 , the corresponding path would start by crossing the ray $\left(\mathbb{R}_{\geqslant 0}, 0\right)$. The path cannot cross the purple wall and it cannot recross the ray $\left(\mathbb{R}_{\geqslant 0}, 0\right)$, because that would correspond to a red mutation. Hence, the path is trapped between the two, and can never reach the all-negative chamber.

Example 32. For $\mathrm{Q}_{1,1,2}$ as in Figure 15a, there are many maximal green sequences, one which can be realized by the path $p$ depicted in Figure 16b.

\footnotetext{
${ }^{10}$ Specifically, there is a bijection between sequences of mutations of a $g$-seed, and finite transverse paths starting in the corresponding reachable chamber up to homotopies in the space of finite transitive paths.

${ }^{11}$ Additionally, it would have to cross an infinite number of walls to get to the purple ray.
} 
The stereographic projection has been chosen so that the point $(1,1,1)$ maps to the origin, and so the green side of the stereographic projection of a wall is always the concave side. A maximal green sequence for $Q_{1,1,2}$ corresponds to a finite transverse path in $\mathfrak{D}\left(\mathrm{Q}_{1,1,2}\right)$ which travels from the inner-most chamber to the exterior, and only crosses walls from the concave side to the convex side.

\section{Proofs}

\subsection{Pullbacks of scattering diagrams}

Let $Q$ be a quiver with vertex set $\{1,2 \ldots, r\}$, and $Q^{\dagger}$ the induced subquiver on the subset $V \subset\{1,2, \ldots, r\}$. How can we describe $\mathfrak{D}\left(\mathrm{Q}^{\dagger}\right)$ in terms of $\mathfrak{D}(\mathrm{Q})$ ?

The inclusion $V \subset\{1,2, \ldots, r\}$ determines a coordinate projection $\pi: \mathbb{R}^{r} \rightarrow \mathbb{R}^{V}$ and coordinate inclusion $\pi^{\top}: \mathbb{R}^{V} \rightarrow \mathbb{R}^{r}$. Define the pullback $\pi^{*}\left(\mathfrak{D}\left(\mathrm{Q}^{\dagger}\right)\right)$ of $\mathfrak{D}\left(\mathrm{Q}^{\dagger}\right)$ to be the scattering diagram for $\mathrm{Q}$ a wall $\left(\pi^{\top}(n), \pi^{-1}(W)\right)$ for each wall $(n, W)$ of $\mathfrak{D}\left(\mathrm{Q}^{\dagger}\right)$.

Our main result is that the pullback of $\mathfrak{D}\left(\mathrm{Q}^{\dagger}\right)$ along the coordinate projection $\pi$ is the reduction of $\mathfrak{D}(\mathrm{Q})$ at the ideal generated by those $y_{k}$ such that $k \notin V$.

Theorem 33. Let $\mathrm{Q}$ be a quiver, let $\mathrm{Q}^{\dagger}$ be the induced subquiver with vertex set $V$, and let $\pi: \mathbb{R}^{r} \rightarrow \mathbb{R}^{V}$ be the projection onto the coordinates in $V$. Then

$$
\pi^{*} \mathfrak{D}\left(\mathrm{Q}^{\dagger}\right)=\mathfrak{D}\left(\mathrm{Q}^{\dagger}\right) /\left\langle y_{i} \mid i \notin V\right\rangle
$$

Proof. For simplicity, we assume $V=\{1,2, \ldots, v\} \subset\{1,2, \ldots, r\}$, and denote elements of $m \in \mathbb{Z}^{r}$ by $m=\left(m_{1}, m_{2}\right) \in \mathbb{Z}^{v} \oplus \mathbb{Z}^{r-v}$. The skew-adjacency matrix decomposes as

$$
\mathrm{B}=\left[\begin{array}{cc}
\mathrm{B}^{\dagger} & -\mathrm{F}^{\top} \\
\mathrm{F} & \mathrm{C}
\end{array}\right]
$$

where $\mathrm{B}^{\dagger}$ is the skew-adjacency matrix of the induced subquiver $\mathrm{Q}^{\dagger}$.

Let $R^{\dagger}:=\mathbb{Z}\left[x_{1}^{ \pm 1}, \ldots, x_{v}^{ \pm 1}\right]\left[\left[y_{1}, \ldots, y_{v}\right]\right]$ and define a ring homomorphism $\phi: R^{\dagger} \rightarrow R$ by

$$
\phi\left(x^{m}\right)=x^{(m, 0)}, \quad \phi\left(y^{n}\right)=x^{(0, \mathrm{~F} n)} y^{(n, 0)}
$$

We check that $\phi$ commutes with the pullback of walls, in that $E_{n} \circ \phi=\phi \circ E_{(n, 0)}$.

$$
\begin{gathered}
E_{(n, 0)}\left(\phi\left(x^{m}\right)\right)=\left(1+x^{\left(\mathrm{B}^{\dagger} n, \mathrm{~F} n\right)} y^{(n, 0)}\right)^{n \cdot m} x^{(m, 0)}=\phi\left(E_{n}\left(x^{m}\right)\right) \\
E_{(n, 0)}\left(\phi\left(y^{n^{\prime}}\right)\right)=E_{(n, 0)}\left(x^{\left(0, \mathrm{~F} n^{\prime}\right)} y^{\left(n^{\prime}, 0\right)}\right)=\phi\left(E_{n}\left(y^{n^{\prime}}\right)\right)
\end{gathered}
$$

We now show that $\pi^{*}\left(\mathfrak{D}\left(\mathrm{Q}^{\dagger}\right)\right)$ is a consistent scattering diagram. Let

$$
I \subset \mathbb{Q}\left[\left[y_{1}, y_{2}, \ldots, y_{r}\right]\right]
$$

be a monomial ideal with finite dimensional quotient, and let $J \subset R^{\dagger}$ be the monomial ideal generated by

$$
\left\{y^{n} \in R^{\dagger} \mid \exists n^{\prime} \in \mathbb{N}^{v-r} \text { s.t. } y^{\left(n, n^{\prime}\right)} \in I\right\}
$$


The induced $R$-ideal $R \otimes_{R^{\dagger}} J$ is the saturation $\left(I:\left\langle y_{i} \mid i \notin V\right\rangle^{\infty}\right)$. Since every wall in $\pi^{*}\left(\mathfrak{D}\left(\mathrm{Q}^{\dagger}\right)\right)$ is of the form $\left((n, 0), \pi^{-1}(W)\right)$ for some $n \in \mathbb{N}^{v}$, the two reductions $\pi^{*}\left(\mathfrak{D}\left(\mathrm{Q}^{\dagger}\right)\right) / I$ and $\pi^{*}\left(\mathfrak{D}\left(\mathrm{Q}^{\dagger}\right)\right) / R \otimes_{R^{\dagger}} J=\pi^{*}\left(\mathfrak{D}\left(\mathrm{Q}^{\dagger}\right) / J\right)$ coincide.

Let $p$ be any transverse loop in $\pi^{*}\left(\mathfrak{D}\left(\mathrm{Q}^{\dagger}\right)\right) / I$, which is necessarily finite. The walls crossed by $p$ are the preimages of the walls crossed by the loop $\pi \circ p$ in $\mathfrak{D}\left(\mathrm{Q}^{\dagger}\right) / J$. Hence, if the path-ordered product of $\pi \circ p$ in $\mathfrak{D}\left(\mathrm{Q}^{\dagger}\right) / J$ is

$$
E_{n_{1}} E_{n_{2}} \cdots E_{n_{k}}
$$

then the path-ordered product of $p$ in $\mathfrak{D}(\mathrm{Q}) / I$ is

$$
E_{\left(n_{1}, 0\right)} E_{\left(n_{2}, 0\right)} \cdots E_{\left(n_{k}, 0\right)}
$$

Any monomial $x^{\left(m_{1}, m_{2}\right)} \in R$ may be written as $\phi\left(x^{m_{1}}\right) x^{\left(0, m_{2}\right)}$, and so

$$
\begin{gathered}
E_{\left(n_{1}, 0\right)} E_{\left(n_{2}, 0\right)} \cdots E_{\left(n_{k}, 0\right)}\left(\phi\left(x^{m_{1}}\right) x^{\left(0, m_{2}\right)}\right)=x^{\left(0, m_{2}\right)} E_{\left(n_{1}, 0\right)} E_{\left(n_{2}, 0\right)} \cdots E_{\left(n_{k}, 0\right)}\left(\phi\left(x^{m_{1}}\right)\right) \\
=x^{\left(0, m_{2}\right)} \phi\left(E_{n_{1}} E_{n_{2}} \cdots E_{n_{k}}\left(x^{m_{1}}\right)\right)=x^{\left(0, m_{2}\right)} \phi\left(x^{m_{1}}\right)=x^{\left(m_{1}, m_{2}\right)}
\end{gathered}
$$

Hence, the path-ordered product of $p$ is trivial on $R / I$, and so $\pi^{*}\left(\mathfrak{D}\left(\mathrm{Q}^{\dagger}\right)\right)$ is consistent.

Both $\pi^{*}\left(\mathfrak{D}\left(\mathrm{Q}^{\dagger}\right)\right)$ and $\mathfrak{D}(\mathrm{Q}) /\left\langle y_{i} \mid i \notin V\right\rangle$ are consistent scattering diagrams in $\mathbb{R}^{r}$ for the matrix $\mathrm{B}$ with the same set of incoming walls $\left\{\left(e_{i}, e_{i}^{\perp}\right) \mid i \in V\right\}$. By the uniqueness theorem [GHKK14, Theorem 1.7], they must coincide.

Remark 34. The scattering diagram $\pi^{*}\left(\mathfrak{D}\left(\mathrm{Q}^{\dagger}\right)\right)$ can be regarded as the scattering diagram of a quiver with frozen vertices; that is, vertices where mutation is prohibited. Here, the quiver is $\mathrm{Q}$ with frozen vertices $V^{c}$. The theorem may be regarded as saying that freezing a vertex $k$ reduces the corresponding scattering diagram by the ideal $\left\langle y_{k}\right\rangle$, and adding a frozen vertex pulls back the corresponding scattering diagram.

Proof of Theorem 9. Given a maximal green sequence on $\mathrm{Q}$, there is a finite transverse path $p$ in $\mathfrak{D}(\mathrm{Q})$ which passes through the corresponding reachable chambers. In particular, $p$ begins in the all-positive chamber and ends in the all-negative chamber. The projection $\pi \circ p$ is a finite transverse path in $\mathfrak{D}\left(\mathrm{Q}^{\dagger}\right)$ which starts in the all-positive chamber and ends in the all-negative chamber, and always crosses walls from the green side to the red side. Hence, the sequence of walls crossed by $\pi \circ p$ determines a maximal green sequence on the induced subquiver $\mathrm{Q}^{\dagger}$.

If a maximal green sequence on $\mathrm{Q}$ begins with a sequence of $k$-many mutations at vertices in $V$, then the path $p$ begins by crossing $k$-many walls in $\pi^{*}\left(\mathfrak{D}\left(\mathrm{Q}^{\dagger}\right)\right) \subset \mathfrak{D}(\mathrm{Q})$. Then the projected path $\pi \circ p$ begins by crossing the images of those $k$-many walls in $\mathfrak{D}\left(\mathrm{Q}^{\dagger}\right)$. The maximal green sequence associated to $\pi \circ p$ then begins with the same $k$ many mutations as the original maximal green sequence.

Proof of Theorem 17. The proof of the analogous result for green-to-red sequences is virtually identical. The only difference is that the corresponding finite transverse paths need not always cross from the green side to the red side.

Remark 35. Once a path $p$ in $\mathfrak{D}(\mathrm{Q})$ from a maximal green sequence crosses a wall not in $\pi^{*}\left(\mathfrak{D}\left(\mathrm{Q}^{\dagger}\right)\right)$, it becomes quite difficult to translate mutations in the original maximal green sequence on $\mathrm{Q}$ into mutations in the induced maximal green sequence on $\mathrm{Q}^{\dagger}$. 


\subsection{Scattering diagrams and mutation}

Given a quiver $\mathrm{Q}$ and its mutation $\mu_{k}(\mathrm{Q})$ at a vertex $k$, there is a natural relation between the corresponding scattering diagrams.

Let $\mathrm{E}_{k}$ be the elementary $r \times r$-matrix with a 1 in the $(k, k)$ entry and 0 s elsewhere, and let $\mathrm{B}^{+}$denote the matrix $r \times r$ matrix with the same positive entries as $\mathrm{B}$ and zero otherwise. Let $\mathrm{B}^{-}:=\mathrm{B}^{+}-\mathrm{B}=\left(\mathrm{B}^{+}\right)^{\top}$. Define a pair of linear maps on $\mathbb{R}^{r}$ as follows.

$$
\mathrm{G}_{k}:=\left(\mathrm{Id}-2 \mathrm{E}_{k}\right)\left(\mathrm{Id}+\mathrm{B}^{+} \mathrm{E}_{k}\right), \quad \mathrm{R}_{k}:=\left(\mathrm{Id}-2 \mathrm{E}_{k}\right)\left(\mathrm{Id}+\mathrm{B}^{-} \mathrm{E}_{k}\right)
$$

These are both involutions which fix the hyperplane $e_{k}^{\perp}$. Their transposes are given by

$$
\mathrm{G}_{k}^{\top}=\left(\mathrm{Id}-2 \mathrm{E}_{k}\right)\left(\mathrm{Id}-\mathrm{E}_{k} \mathrm{~B}^{-}\right), \quad \mathrm{R}_{k}^{\top}=\left(\mathrm{Id}-2 \mathrm{E}_{k}\right)\left(\mathrm{Id}-\mathrm{E}_{k} \mathrm{~B}^{+}\right)
$$

The following lemma describes how the scattering diagram $\mathfrak{D}(\mathrm{Q})$ changes when $\mathrm{mu}$ tating a vertex in $\mathrm{Q}$.

Lemma 36. [GHKK14, Theorem 1.33] Given a quiver $\mathrm{Q}$ and a vertex $k$, the scattering diagram $\mathfrak{D}\left(\mu_{k}(\mathbf{Q})\right)$ is equivalent to the scattering diagram $\mathfrak{D}^{\prime}$ constructed as follows.

- For each $(n, W) \in \mathfrak{D}$, if $W_{+}:=\left\{w \in W \mid e_{k} \cdot w \geqslant 0\right\}$ spans a hyperplane, then

$$
\left(\mathrm{G}_{k}^{\top}(n), \mathrm{G}_{k}\left(W_{+}\right)\right)
$$

is a wall in $\mathfrak{D}^{\prime}$.

- For each $(n, W) \in \mathfrak{D}$, if $W_{-}:=\left\{w \in W \mid e_{k} \cdot w \leqslant 0\right\}$ spans a hyperplane, then

$$
\left(\mathrm{R}_{k}^{\top}(n), \mathrm{R}_{k}\left(W_{-}\right)\right)
$$

is a wall in $\mathfrak{D}^{\prime}$.

- For each $(n, W) \in \mathfrak{D}$, if $W$ is contained in $e_{k}^{\perp}$, then $(n, W)$ also a wall in $\mathfrak{D}^{\prime}$.

As a corollary, the piece-wise linear map

$$
T_{k}: m \mapsto\left\{\begin{array}{ll}
\mathrm{G}_{k}(m) & \text { if } e_{k} \cdot m \geqslant 0 \\
\mathrm{R}_{k}(m) & \text { if } e_{k} \cdot m \leqslant 0
\end{array}\right\}=\left\{\begin{array}{ll}
\left(\mathrm{Id}-2 \mathrm{E}_{k}\right)\left(\mathrm{Id}+\mathrm{B}^{+} \mathrm{E}_{k}\right) m & \text { if } e_{k} \cdot m \geqslant 0 \\
\left(\mathrm{Id}-2 \mathrm{E}_{k}\right)\left(\mathrm{Id}+\mathrm{B}^{-} \mathrm{E}_{k}\right) m & \text { if } e_{k} \cdot m \leqslant 0
\end{array}\right\}
$$

sends the support of walls in $\mathfrak{D}(\mathrm{Q})$ to the support of walls in $Q\left(\mu_{k}(\mathrm{Q})\right)$, and thus chambers of $\mathfrak{D}(\mathrm{Q})$ to chambers of $\mathfrak{D}\left(\mu_{k}(\mathrm{Q})\right)$.

Remark 37. The construction of $\mathfrak{D}^{\prime} \simeq \mathfrak{D}\left(\mu_{k}(\mathrm{Q})\right)$ appears different in [GHKK14], because they consider scattering diagrams with normal vectors $n$ in a simplicial cone $P \subset \mathbb{Z}^{r}$, rather than $\mathbb{N}^{r}$. Composing the construction in [GHKK14] with a linear map which takes their $P$ to $\mathbb{N}^{r}$ recovers the above construction of $\mathfrak{D}^{\prime}$. 
Proof of Theorem 18. First, we consider the conjugation of a green-to-red sequence by a single mutation of $\mathrm{Q}$ at vertex $k$.

A green-to-red sequence on $\mathrm{Q}$ corresponds to a finite transverse path $p$ in $\mathfrak{D}(\mathrm{Q})$ which begins in the all-positive chamber and ends in the all-negative chamber. The image $T_{k} \circ p$ is a finite transitive path in $\mathfrak{D}\left(\mu_{k}(\mathrm{Q})\right)$. The path $T_{k} \circ p$ begins in the chamber which shares the wall $\left(e_{k}, e_{k}^{\perp}\right)$ with the all-positive chamber and ends in the chamber which shares the wall $\left(e_{k}, e_{k}^{\perp}\right)$ with the all-negative chamber. Choose a path $p_{1}$ from the allpositive chamber to the beginning of $T_{k} \circ p$ which only crosses $\left(e_{k}, e_{k}^{\perp}\right)$ once and does not touch any other wall. Similarly, choose a path $p_{2}$ from the end of $T_{k} \circ p$ to the all-negative chamber which only crosses $\left(e_{k}, e_{k}^{\perp}\right)$ once and does not touch any other wall.

There is a finite transitive path which travels along $p_{1}$, then travels along $T_{k} \circ p$, and then travels along $p_{2} \cdot{ }^{12}$ This path begins in the all-positive chamber and ends in the all-negative chamber, so it determines a green-to-red sequence on $\mathrm{Q}^{\dagger}$. Specifically, the sequence of mutations it determines is the conjugation of the original green-to-red sequence.

By iterating this argument for an arbitrary sequence of mutations on $Q$, the general theorem is proven.

\subsection{Visualizing $\mathfrak{D}\left(\mathbf{Q}_{2,2,3}\right)$}

We can now deduce enough about the scattering diagram $\mathfrak{D}\left(\mathrm{Q}_{2,2,3}\right)$ to visualize why there are no maximal green sequences for $Q_{2,2,3}$, without having to compute the entire scattering diagram. As in Examples 24 and 28, scattering diagrams in $\mathbb{R}^{3}$ may be visualized by intersecting with a unit sphere and stereographically projecting onto the plane $x_{1}+x_{2}+$ $x_{3}=\sqrt{3}$.

For any vertex $i$, the reduced scattering diagram $\mathfrak{D}\left(\mathrm{Q}_{2,2,3}\right) /\left\langle y_{i}\right\rangle$ is the pullback of the scattering diagram of a quiver with 2 vertices. These three reductions of $\mathfrak{D}\left(\mathrm{Q}_{2,2,3}\right)$ are depicted in Figure 17. Each reduction contains a half-plane which supports an infinite number of walls; these are purple in the figure (the second reduction has an infinite number of half-planes which support an infinite number of walls; these are dense in the purple region).

The stereographic projection has been chosen so that the point $(1,1,1)$ maps to the origin, and so the green side of the stereographic projection of a wall is always the concave side. A maximal green sequence for $\mathrm{Q}_{2,2,3}$ corresponds to a finite transverse path in $\mathfrak{D}\left(\mathrm{Q}_{2,2,3}\right)$ which travels from the inner-most chamber to the exterior, and only crosses walls from the concave side to the convex side.

Consider a finite transverse path which starts in the inner-most chamber, and only crosses walls from the concave side to the convex side. As soon as it crosses a wall, the path is now trapped between the convex side of the wall it just crossed (which is a coordinate hyperplane, and hence a circle in the projection) and a purple half-space. Hence, it can never reach the exterior chamber, and so a maximal green sequence cannot exist.

\footnotetext{
${ }^{12}$ Some smoothing may be required at the meeting points between paths.
} 

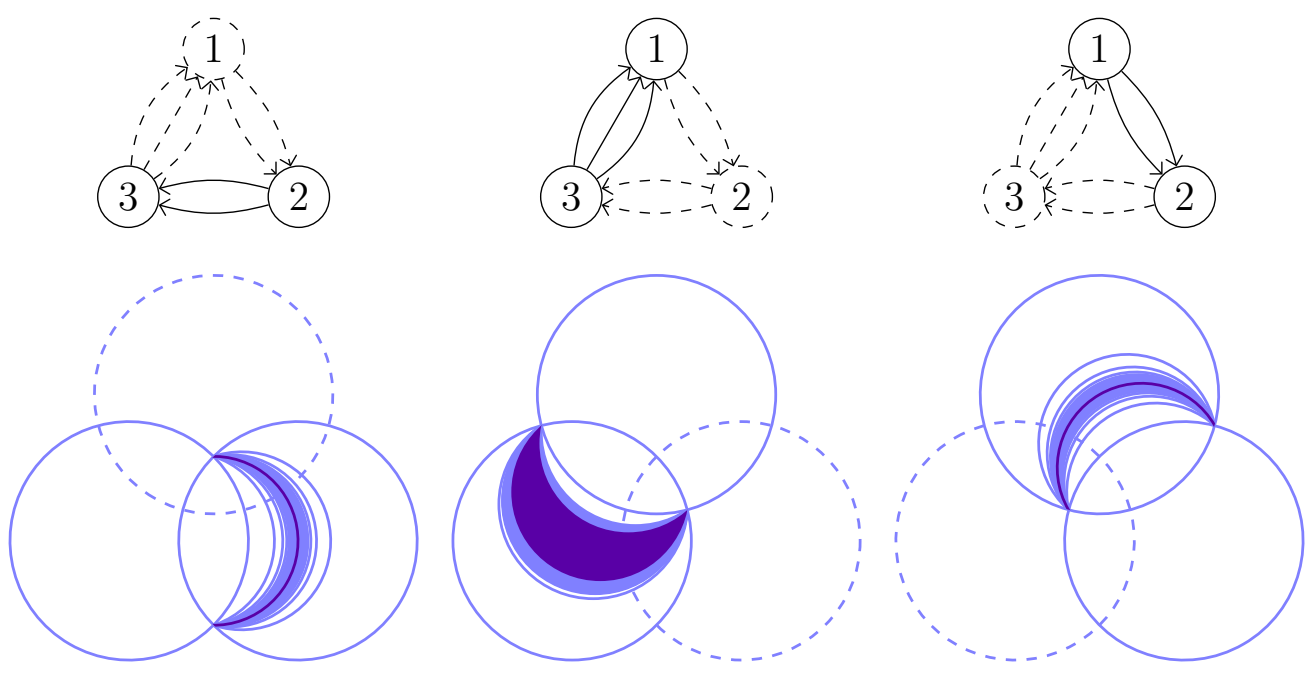

Figure 17: Stereographic projection of three reductions of $\mathfrak{D}\left(\mathrm{Q}_{2,2,3}\right)$

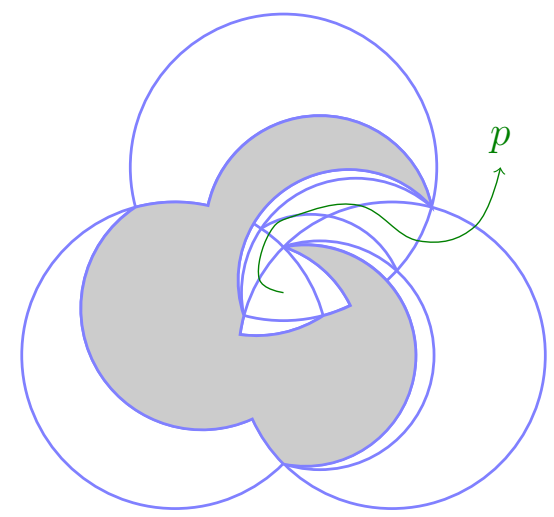

Figure 18: Stereographic projection of 16 chambers of the scattering diagram $\mathfrak{D}\left(\mathrm{Q}_{2,2,3}\right)$, and the path of a green-to-red sequence

Figure 18 draws a piece of $\mathfrak{D}\left(Q_{2,2,3}\right)$; the grey denotes regions where the wall structure has not been computed. Note the existence of a finite transverse path $p$ connecting the inner-most chamber to the exterior chamber; this corresponds to the green-to-red sequence in Figure 11.

\section{Acknowledgements}

The author is indebted to various lectures and conversations with Mark Gross, Sean Keel, and Paul Hacking about their work. This work was inspired by the AMS Mathematics Research Community workshop on Cluster Algebras in 2014, and the author benefited from conversations with the many participants there. The author is also grateful to the anonymous referee for thoughtful feedback. 


\section{References}

[ACC $\left.{ }^{+} 13\right]$ Murad Alim, Sergio Cecotti, Clay Córdova, Sam Espahbodi, Ashwin Rastogi, and Cumrun Vafa. BPS quivers and spectra of complete $N=2$ quantum field theories. Comm. Math. Phys., 323(3):1185-1227, 2013.

[BBH11] Andre Beineke, Thomas Brüstle, and Lutz Hille. Cluster-cyclic quivers with three vertices and the Markov equation. Algebr. Represent. Theory, 14(1):97112, 2011. With an appendix by Otto Kerner.

[BDP14] Thomas Brüstle, Grégoire Dupont, and Matthieu Pérotin. On maximal green sequences. Int. Math. Res. Not., 2014(16), 4547-4586

[Buc14] Eric Bucher. Maximal green sequences for cluster algebras associated to the n-torus. 12 2014, arXiv:1412.3713.

[DWZ10] Harm Derksen, Jerzy Weyman, and Andrei Zelevinsky. Quivers with potentials and their representations II: applications to cluster algebras. J. Amer. Math. Soc., 23(3):749-790, 2010.

[FZ02] Sergey Fomin and Andrei Zelevinsky. Cluster algebras. I. Foundations. J. Amer. Math. Soc., 15(2):497-529 (electronic), 2002.

[FZ07] Sergey Fomin and Andrei Zelevinsky. Cluster algebras. IV. Coefficients. Compos. Math., 143(1):112-164, 2007.

[GHK15] Mark Gross, Paul Hacking, and Sean Keel. Mirror symmetry for log CalabiYau surfaces I. Publ. Math. Inst. Hautes Études Sci., 2015(122), 65-168, 2015

[GHKK14] Mark Gross, Paul Hacking, Sean Keel, and Maxim Kontsevich. Canonical bases for cluster algebras. 11 2014, arXiv:1411.1394.

[GMN10] Davide Gaiotto, Gregory W. Moore, and Andrew Neitzke. Four-dimensional wall-crossing via three-dimensional field theory. Comm. Math. Phys., 299(1):163-224, 2010.

[GPS10] Mark Gross, Rahul Pandharipande, and Bernd Siebert. The tropical vertex. Duke Math. J., 153(2):297-362, 2010.

[Kel11] Bernhard Keller. On cluster theory and quantum dilogarithm identities. In Representations of algebras and related topics, EMS Ser. Congr. Rep., pages 85-116. Eur. Math. Soc., Zürich, 2011.

[KS11] Maxim Kontsevich and Yan Soibelman. Cohomological Hall algebra, exponential Hodge structures and motivic Donaldson-Thomas invariants. Commun. Number Theory Phys., 5(2):231-352, 2011.

[KS13] Maxim Kontsevich and Yan Soibelman. Wall-crossing structures in Donaldson-Thomas invariants, integrable systems and Mirror Symmetry. 03 2013, arXiv: 1303.3253.

[Pla11] Pierre-Guy Plamondon. Cluster characters for cluster categories with infinitedimensional morphism spaces. Adv. Math., 227(1):1-39, 2011. 
[Rei10] Markus Reineke. Poisson automorphisms and quiver moduli. J. Inst. Math. Jussieu, 9(3):653-667, 2010.

[Sev14] Ahmet I. Seven. Maximal green sequences of exceptional finite mutation type quivers. SIGMA 10 (2014), 089, 5 pages, 06 2014, arXiv:1406.1072.

[Yak] M. Yakimov. Maximal green sequences for double Bruhat cells. In preparation. 